\title{
Context and Meaning: The Challenges of Metadata for a Digital Image Library within the University
}

\author{
John Attig, Ann Copeland, and Michael Pelikan
}

\begin{abstract}
To be successful in the university environment, a digital library must be able to integrate content from faculty and students, as well as traditional library sources. It must have a robust metadata structure that can accommodate and preserve a variety of discipline-specific metadata while supporting consistent access across collections. As part of the Mellon-funded project, the Visual Image User Study at Penn State, a prototype centralized digital image delivery service was created and explored. In creating a metadata schema for the project, the authors anticipated both a wide variety of content and users across many disciplines. This schema employed three very different standards (VRA Core Categories, Dublin Core, IMS Learning Objects Meta-data). The project validated the need for highly individualized content, the importance of individual faculty collections, the need for editorial intervention to supplement and modify contributed metadata, and the importance of addressing discipline-specific vocabularies and taxonomies.
\end{abstract}

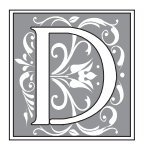

igital libraries are developed within a context, and that context determines the character of the design. Most digital libraries are organized and administered by libraries, but many exist within a university system. In this context, the teaching and research functions of the university are determining factors. Members of the university community are rapidly becoming accustomed to using digital objects in their teaching and research. They have their own content (both digital and not-yet-digital), and they seek additional content relevant to their work. Faculty members are also likely to be users of university course management systems that provide an electronic environment for the classroom experience and which make digital learning objects available to their students. If a digital library is to be successful in this environment, it must integrate these sources of content and these systems into its design.

This paper seeks to validate one aspect of this argument-the design of an appropriate metadata infrastructure-based on the authors' participation in a project to study the needs of users of images in an academic environment. As part of this

John Attig is Monograph Cataloging Librarian/Authority Control Librarian and Associate Librarian; e-mail: jxa16@psulias.psu.edu; Ann Copeland is Special Collections Cataloging Librarian and Assistant Librarian; e-mail:auc1@psulias.psu.edu; and Michael Pelikan is Technology Initiatives Librarian and Assistant Librarian; e-mail: mpp10@psulias.psu.edu; all at Pennsylvania State University Libraries. 
study, a prototype digital image library was developed and assessed. This prototype was to include a robust metadata schema appropriate to the content. This effort was interesting, as the authors tried to reconcile some strikingly diverse metadata standards. Even more interesting were the challenges the authors faced in applying these schemas to the actual digital content selected for the prototype image library.

\section{Background and Description of the Visual Image User Study (VIUS)}

The provision of digital images for higher education has been an active area for research, design, and production projects in recent years. Many institutions have digitized collections and built institutional repositories, and continue to do so. Vendors are rapidly developing software to assist them in those endeavors. Products such as Luna's Insight Software, James Madison's MDID, and OCLC's contentDM enable the establishment of collections, addition of content, organization of images, and creation of metadata. Research projects during the 1990s, such as the Museum Educational Site Licensing Project (MESL) and the AMICO Library and Testbed, seemed to take a holistic approach, exploring production (multi-institutional cooperative production, in these cases), metadata coordination, system design, and user study (usually evaluating the results of the other efforts).$^{1,2}$ Research projects that followed these two important precedents have tended to focus on specific aspects of these problems. An NEH-sponsored grant project at the Rochester Institute of Technology's Image Permanence Institute focused on the technical aspects of digitization. ${ }^{3}$ The UCAI (Union Catalog of Art Images) project at UCSD has explored the problem of coordinating metadata standards in a way that will facilitate shared cataloging of images. ${ }^{4,5}$ The Visual Image
User Study (VIUS) at Penn State was unusual among these research projects for taking a user-first approach, starting with a study of existing user habits and preferences to inform an approach to questions of metadata design, system features, and so on. ${ }^{6}$

Penn State University received a generous grant from the Andrew W. Mellon Foundation in 2001 to conduct a 26-month project to assess the requirements for a digital image delivery system. The grant application grew out of discussions within the University Libraries about the scope, nature, and content of the digital library. The opportunity to examine one significant category of content-still images-as an aspect of digital library development immediately drew willing partners from outside the libraries, including museums, the Center for Education Technology Services, the School of Information Sciences and Technology, and faculty members in several departments. Together with the authors, they sought to understand how images are currently used on campus, what functions are supported by image use, who uses them, and the degree to which image use has become digital.

The first phase of the VIUS focused on the study of user needs through focus groups, surveys, protocol testing, authentication log analysis, and other data collection methods. The second phase involved the construction of two prototype image delivery systems: a centralized set of collections administered by the libraries and a peer-to-peer system supporting the sharing of digital objects in an authenticated environment (still in development). The final phase comprised the evaluation of these prototype services for usability, user satisfaction, and sustainability.

The study yielded extremely useful data, which may be found in the final report on the VIUS Web site. Some of the key findings provide the background to 
the argument above about the design of digital libraries within an academic environment, as well as the assumptions that governed the design of a metadata infrastructure for the prototype database. Briefly, these findings are as follows:

- There is considerable interest among faculty and students in using images for teaching and research $(75 \%$ of faculty and $55 \%$ of students who responded to the survey currently use images). A considerable number already maintain individual picture collections for professional reasons ( $44 \%$ of faculty and $44 \%$ of students say they have personal collections). A smaller, but significant, number are using digital images $(50 \%$ of faculty and $45 \%$ of students), and many more expect to increase their use of digital images in the next three years (75\% of both faculty and students).

- Potential users of a digital image library (faculty members, in particular) are more concerned about content-related issues than they are about retrieval-related issues; they are less concerned with how to discover images than with whether the image library will contain relevant images at all. Both their teaching and research needs tend to be highly individualized.

- Many potential users are already building collections of images. The fact that they have specifically selected and retained these images to meet their requirements means that these collections are a critical component that needs to be incorporated within a digital library.

- Faculty members are ambivalent about sharing their collections but are interested in the possibilities for collaboration and for shared development of core image collections.

The study focused specifically on the arts, humanities, and environmental studies for the purposes of the user study but anticipated that the production service to follow would contain collections and images across an even greater range of disciplines. The prototype centralized image library, on the other hand, was limited to art and architecture. Digital content was obtained from a variety of sources: commercial images, faculty collections, material from library book and archival collections, departmental and museum collections. One specific goal was to build a core collection of images to support the teaching of an introductory survey of landscape architecture.

\section{Metadata}

The function of descriptive metadata is to support discovery of appropriate resources. Because digital objects are created by specific user communities (and even individuals) with their own needs and vocabularies, the context in which a digital object originates has considerable bearing on what type of descriptive metadata is appropriate to the object. The metadata about Notre Dame Cathedral necessarily would be different from that for a botanical specimen. Categories such as date, building name, and type of building would be relevant for the former while the scientific name and taxonomy would be appropriate for the latter.

Tony Gill has used the term "cultural infodiversity" to express the necessarily heterogeneous nature of cultural information, with the conclusion that "no single (meta)data schema fits all." Although some descriptions are simple and generic, others are richer and domain specific. Access into these descriptions is often through the lowest common denominator provided by a crosswalk, ultimately leading the metadata editor into "mapping madness." ${ }^{8}$ In the case of a digital library, with collections brought together from multiple disciplines and diverse sources, the digital images are both "infodiverse" and varied in terms of appropriate descriptive information. Clearly no single metadata standard 
would be sufficient. The authors decided immediately to engage in their own exercise in "mapping madness" in order to accommodate different descriptive metadata standards in a way that would support their interoperation. Given the focus of the study on the use of images in an academic environment, The authors quickly focused their attention on three emerging descriptive metadata standards: VRA Core, Dublin Core, and IMS Learning Resource Meta-data.

\section{VRA Core}

The focus on images and on the disciplines of art and architecture for the prototype suggested that one standard that had to be accommodated was the Core Categories developed by the Visual Resources Association (VRA). The VRA Core consists of a single element set, with elements that may be repeated as many times as necessary to describe works of visual culture as well as the images that document those works. The distinction between the works and the visual images is a unique feature of the VRA Core. In an ideal system, a separate description would be created for each work (e.g., an individual building) and for each image of that work (e.g., a plan of the building or a photograph of the façade); each description would include the element Record Type containing either the value "work" or the value "image." The element set includes elements specific to the discipline of art history, such as Style/Period. Qualifiers also are suggested for some elements to provide more detailed semantics.

\section{Dublin Core}

Given that the potential digital library would need to encompass all disciplines, a common metadata language- Gill's "lowest common denominator" - would most likely be needed. This suggested that the Dublin Core Metadata Element
Set (DC) should be examined. DC is a set of fifteen descriptive elements designed to provide a simple means for describing resources to aid discovery and retrieval. Optionally, qualifiers may be used for a variety of purposes: to refine the semantics of an element (e.g., distinguishing a date of creation from a date of modification) or to identify the source of the data content (e.g., the use of the ISO standard for dates or of terms from a particular thesaurus).

\section{IMS Learning Resource Meta-data}

The authors' partnership with the campus instructional technology center strongly suggested that the IEEE Learning Object Meta-data (LOM) 6-1 / IMS Meta-data Specification 1.2.1 (hereafter referred to as IMS Learning Resource Meta-data) also be considered. ${ }^{9}$ Penn State is actively using ANGEL, a learning management system that facilitates course instruction and enables reviews, quizzing, and chat (i.e., the delivery of various digital learning objects). Further, the Center for Educational Technology Services is engaged in a variety of projects involving collaborative learning management supported by the IMS Learning Resource meta-data standard..$^{10}$ The purpose of the standard is to facilitate search, evaluation, acquisition, and use of learning objects, by learners or instructors, and to facilitate the sharing of learning objects by developing and exchanging catalogs and inventories of learning objects. The standard attempts to take into account the diversity of cultural and linguistic contexts in which learning objects and their metadata will be created and exploited. ${ }^{11}$ This emerging standard is not wholly new to libraries but has not yet been adopted widely or reckoned with in the design of digital libraries. The standard contains a number of unique features, perhaps most significantly its deeply hierarchical structure. It contains data elements appropriate to learning 
objects and their use. For example, the element LearningResourceType would be used for such objects as exercises, simulations, questionnaires, and exams.

\section{Metadata Structures: Challenges and Realities}

The basic assumption of the authors' metadata design for the VIUS prototype database was that each of these three metadata standards needed to be supported, that images included in the database might include metadata in any one or more of these standards, that the specificity of the metadata should be preserved in the VIUS database, and that some sort of mapping would be required to support searching across the entire database.

Given these objectives, it was decided to create a "merged superset" of all the elements in the three standards. Because the standards have been created to meet the needs of specific communities, the authors wanted to preserve the context and specificity of the metadata created within each standard, with no loss of data in the process of merging.

Thus, a schema was created that incorporates all possible elements that might be carried by metadata contributed to the system. The schema provides an element that corresponds to each element in the three standards, allowing the specificity of any imported metadata to be preserved. Where appropriate, the schema identifies equivalent elements across the three standards; these tend to correspond roughly to elements in the Dublin Core, and the hope was that these common elements would be sufficient to support searching across the database. The schema, which could be expressed as an XML schema or DTD, would eventually support data transformations for importing and exporting data, for generating customized displays, and for creating a common query structure. The schema, in tabular form, is available on the VIUS Web site. ${ }^{12}$

The basic mapping was not terribly difficult. Many of the elements in each of the standards either had nearly equivalent definitions or were specific cases of elements in other standards. However, a closer examination of the mappings revealed difficulties. As the IMS "best practices" Web site states: "It is important to note that just because it is possible to map Dublin Core and IMS Learning Resource Meta-data elements to each other, this does not mean the elements are semantically or structurally equivalent. The reader should carefully study and understand both the meaning and intended usage of each element before utilizing it in a meta-data record."13

The authors found this warning to be amply warranted. There were semantic differences between the definitions of superficially similar data elements. Data elements using terms such as "type" or "format," for example, were very likely to be intended to have quite different data values and might even have different functional uses. Another problematic set of elements are those that specify the "subject" coverage. Not surprisingly, the key elements of subject specification vary considerably from discipline to discipline. The VRA element Style/Period is a good example of a highly specific element; it is somehow related to the DC elements Subject or Coverage.Chronological but cannot be reduced to either DC element without significant loss of meaning. ${ }^{14}$

In addition to semantic problems, structural differences between the standards presented a significant challenge. Moving between a profoundly hierarchical standard such as the IMS Learning Resource Meta-data standard and a flat (albeit potentially qualified) set of elements such as DC was difficult to model. For example, the straightforward DC 
element Subject is represented in IMS as Classificiation.TaxonPath.Taxon.Entry. ${ }^{15}$ Translating the two-level VRA structure (every element applies to either the work or the image) into standards that do not support this structure was impossible. The place of an element in a hierarchical structure serves to express some of its meaning. That context must not be lost when mapped to a different structure; it is unclear to what extent this is possible.

The authors concluded that their merged superset was an important feature of their design because it allowed the preservation of that context. It also meant that data transformations based on mapping between elements could be accomplished only at considerable risk of loss of meaning and should never be permanent transformations of the stored metadata. The critical question is whether the limited number of truly equivalent elements would be sufficient to support searching across the database.

The mapping of data elements is comparatively straightforward. However, the manipulation of data between elements by itself will not be sufficient to make the data truly interoperable. The main difficulties concern the meaning of the values contained in the elements. There are many sources for these difficulties. They may arise out of contextual differences in the use of language in different disciplines or differences in the role that the data element itself plays in imparting meaning to the values (the hierarchical context). Regardless of the source of the differences, mapping is about meaning. Anything that contributes to meaning in one context must be mapped into the new context or there will be loss of meaning.

Unfortunately, it was not possible to test the authors' metadata schema in the prototype. The need to select an available software platform for the prototype imposed limitations on data structure, and the schema could not be accommodated. However, the authors feel that their approach was both valid and appropriate, and hope to test it in some other context.

\section{Metadata Values}

The difficulties that can arise in mappings between even the most carefully defined elements in published metadata specifications have already been mentioned. Further complications arise given the variability of the values entered into elements. When speaking about values, the authors are talking about the content within a descriptive element, the term entered therein. If the element is Date, for example, the string "2003" would be a value. However, several factors come into play with the assignment of values, for example, the ambiguity of language, the subjectivity of individual collections, the subjectivity of persons describing the content, and the heterogeneous nature of disciplines and cultures that inform description. These factors are particularly significant in the university environment in which both faculty and students tend to work within particular disciplines, each with their own worldview, complete with vocabularies, taxonomies, and interests. The university, as a summation of these specific disciplines, must both nurture the specificity and support interdisciplinary serendipity.

One word may mean different things. For instance, the word bridge may mean one thing to an engineer, another to a dentist, and quite another to a violinist. This reveals the relative sway that our disciplinary homes can have in informing our choices. Beyond this, just as the same words can mean different things, many words mean similar things. What one person calls the "bridge" between sections of a musical score, for example, another person might call a "transition." Thus, the same object may be described in many ways. How is it possible to ensure that consistent 
terminology is employed for consistent values in describing an object?

Further, as humans, our perspective and focus are unique. For example, a photograph taken in Venice might show a famous cathedral on a square with pigeons, a canal, and several boats wending their way under a bridge. What "defines" this scene? What descriptive terms might it suggest, and how would they be assigned? It seems clear that someone looking at the scene would identify the elements it contains in the same terms that they would use in retrieving the image. How a person chooses to retrieve the image will influence how he or she assigns descriptive terms to it. The architectural historian may well assign a "name" to the scene based on the cathedral's name. The structural engineer might label the scene "bridge," and the ornithologist might assign the name Columba fasciata. Clearly none of these terms, in and of themselves, defines the scene. At the same time, assignment of the label "Venice" does nothing to assist the searcher who is looking for pigeons.

One can foresee the need for a system to permit the attachment of multiple, discipline-centric descriptive records to images. Such a system would permit one to choose to search the resource employing the vocabulary of the art historian, for example, or the structural engineer, or the ornithologist. ${ }^{16}$

For now, problems with data values are best dealt with using authority control and content standards. In a published metadata specification, carefully defined elements are fixed and their design and structure are described in the standard's Information Model. Accompanying the Information Model should be a Best Practices Guide, in which the designers of the standard attempt to guide real-world users in its proper application. Absent a specific set of rules or practices govern- ing the choice of values to be entered, or a controlled vocabulary from which to choose the terms (and a determination to employ the rules or the vocabulary or both), even the most rigorously defined elements cannot control the vagaries of humans and free association. To ensure interoperability and consistency among terms and elements, such problems are best countered by authority control and content standards.

Functionally, this could be accomplished using a toolkit that could be customized for a user's disciplinary worldview and would include guidance on selecting data elements and vocabulary. Such a toolkit could consist of Web-based forms, with blanks to be filled in and dropdown lists of suggested terms. This would encourage user contributions, preserve disciplinary understandings of terms, and maintain consistency among users.

\section{Contexts and Boundaries: Some Questions for the Future}

Experienced librarians and seasoned catalogers face numerous challenges in combining the worldviews of descriptive standards. What are the functional requirements for a system that would mediate these issues in a way that is simple, transparent, or invisible to the end user?

In the end, the authors found their elaborate metadata schema of limited use. They discovered that available images usually come with little descriptive metadata. Faculty collectors might be able to supply a caption or title for each image (although sometimes this was a separate list with uncertain connection to the actual images). The authors were more surprised at the limited metadata that could be obtained from image vendors. This reality significantly shifted the focus of the study. Instead of asking how to preserve complex metadata, the authors 
were forced to ask how little metadata would be required for discovery. This question is particularly important for image data. With text, inherited metadata could be mined, as could the content of the resource itself. With visual images, on the other hand, there is almost nothing in the resource itself that can be mined. ${ }^{17}$

One of the findings of the VIUS project is that individual collections are a significant component of a digital library and users therefore need to be able to add images dynamically, share images with colleagues, and merge their own images with those contributed to the database from other sources. This in turn requires that users be able to contribute metadata along with the images. In the authors' plans, this would be accomplished technically by providing a data input form with element names appropriate to the user's disciplinary area. These elements could be mapped to appropriate elements in a generic interchange standard such as Dublin Core. The input forms for various disciplines could be prebuilt, with mappings accomplished. Such a standard would itself have to be discipline agnostic, while providing a machine-understandable means of conveying the contextual aspects of the record set needed to render its meaning accurately. A system such as this would have to deal properly with an aggregated set of records drawn from heterogeneous contexts. At the same time, it would have to enable interoperability among combined sets of records drawn from other similar (or sufficiently similar) contexts. The enabling lynchpin of such a system would be the establishment of a machine-readable standard for anchoring small text-based record sets containing vocabulary terms to subject- or contextspecific controlled vocabularies.

However, the addition of unmediated metadata raises issues apart from the mechanical, technical aspects of the transformations. Thus, again, how little information can be required without endangering the ability to retrieve the images? The requirements need to be kept modest if contributors are expected to provide the metadata. It also is important to consider how much oversight (i.e., manual editing) is needed to make up for either insufficient data or inconsistent data content. These issues need to be resolved if user-contributed metadata is to coexist with more complete, more consistent metadata.

There have been notable efforts to provide a technological framework in which to focus the still-inescapable human effort required to address these issues. The W3C Semantic Web exemplifies such efforts. W3C's introductory explanation for its Semantic Web effort speaks to the need for "having data on the web defined and linked in a way that it can be used by machines not just for display purposes, but for automation, integration and reuse of data across various applications." ${ }^{18}$ It is useful to remember that although such linkages are maintained in a machine environment, their meaning relies on human attention to their appropriate application.

Another approach can be found in the IMS Vocabulary Definitions Exchange (VDEX) specification, in public draft as of this writing. VDEX "defines a grammar for the exchange of simple machine-readable lists of values, or terms, together with information that may aid a human being in understanding the meaning or applicability of the various terms." ${ }^{19}$ VDEX has potential applicability in the exchange of thesaurus fragments that could be unambiguously combined from multiple repositories. The contextual aspects thus captured could include the disciplinary context (by identifying the controlled vocabulary in use), as well as the specific thesaurus relationships among those terms expressed in machinereadable form. Here again, the technology 
provides a framework for the interchange of values and their relation to each other, but the system supports, rather than supplants, the human effort. The discernment of subtle, but crucial, differences in meaning will likely forever elude brute force or the blunt instrument.

\section{Conclusion}

If a digital image library - or indeed any digital library collection-is to be successful within a university environment, it must accommodate the highly specific content that faculty and students are using in their teaching and research. Such a digital library must be able to integrate content from various sources: existing library collections, individual faculty and student collections, content vendors, and other institutions. At the foundation of such a system must be a robust metadata schema capable of both supporting the needs of all the disciplines that comprise the university and interoperating with learning management systems throughout the university, transporting images and learning objects to the classroom, a dorm room, or a customized individual collection in response to user queries.

The authors' analysis of these metadata requirements caused them to construct a merged superset of several descriptive metadata standards. This was done in order to maintain disciplinary data elements and vocabulary, while facilitating resource discovery across diverse collections. The authors' schema does allow for the preservation of metadata from each standard in all its specificity. The corresponding argument that it supports a sufficient level of interoperability has not been tested in application. However, similar schemas are being developed for other digital projects, and with their implementation will come real-world assessment. Over time, clarity should emerge regarding the issues raised by elements and their mapping in relation to context and its meaning for the values contained in those mapped elements.

Another unavoidable issue concerns the limited metadata that generally comes with a digital object. Here a variety of solutions are needed. Active contributors of digital objects need to be provided with a toolkit for contributing metadata that is customized for their disciplinary worldview. Metadata obtained from outside sources (vendors, other institutions) may need to be edited manually. In both cases, authority control and content guidelines are needed to ensure consistency.

University faculty and students are involved in the creation of content and are actively seeking additional content. A successful digital library program must integrate the content they create and provide with the highly individualized content that they seek. Metadata is the key to this endeavor. The institution must invest in the creation of appropriate standards and practices for acquiring and integrating content and its associated metadata; it must invest human resources in establishing these standards and practices as well as in their consistent application. The guiding principles should be to preserve the disciplinary context of the digital objects, but also to allow them to be retrieved in a centralized system supporting a multidisciplinary universe.

The authors have argued in favor of a merged superset of descriptive standards that would allow for maintaining disciplinary relationships while facilitating cross-collection searching and discovery. They believe that their schema would allow for the preservation of disciplinespecific descriptive metadata. Cognizant of the dynamic nature of the enterprise, the authors recognize that contributors to such an endeavor will need to adhere to modest metadata requirements, customized for their disciplinary worldviews. 


\section{Notes}

1. The Museum Educational Site Licensing Project (MESL), an initiative of the Getty Art History Information Project, was a two-year collaborative research effort among seven museums and seven universities to share digital images.

2. The AMICO Library and Testbed was a use and evaluation study of a collection of more than 20,000 documented, digitized images contributed by major art museums for licensed use by institutions of higher education. Available online from www.rlg.org/amicolib.html. [Accessed 30 January 2004].

3. The report from 1999 identifies the key issues affecting image quality and explores ways to measure digital image quality. Available on the IPI Web site at http://www.rit.edu/ipi. [Accessed 30 January 2004].

4. UCAI (Union Catalog of Art Images) is available online from http://gort.ucsd.edu/ucai/. [Accessed 30 January 2004].

5. ARTstor, a digital repository of content in the history of art provided by various institutions, seems to draw from many of these research and production projects to provide a significant new digital image delivery service. Available online from http://www.artstor.org. [Accessed 30 January 2004].

6. The Visual Image User Study is available online from http://www.libraries.psu.edu/vius/ reports.html. [Accessed 11 November 2003].

7. Tony Gill, "Is That a Reference Model in Your Pocket? The CIDOC CRM \& the IFLA FRBR," PowerPoint presentation from Ready to Wear: Metadata Standards to Suit Your Project, An RLGCIMI Forum, 12-13 May 2003. Available online from http://www.rlg.org/events/metadata2003/gill. ppt. [Accessed 11 November 2003].

8. Ibid.

9. In explanatory text intended to clarify the relation between the IEEE Learning Object Meta-Data (LOM) Draft Standard and IMS Learning Resource Meta-data, the IMS Global Learning Consortium notes on its Web site;," The IEEE LOM Draft Standard defines a set of meta-data elements that can be used to describe learning resources. This includes the element names, definitions, data types, and field lengths. The specification also defines a conceptual structure for the meta-data.

The IEEE LOM Draft Standard is intended to support consistent definition of meta-data elements across multiple implementations, but does not (at the time of this writing) include information on how to represent meta-data in a machine-readable format, necessary for exchanging meta-data. The IMS developed a representation of the meta-data in XML (eXensible Markup Language).

The IMS Learning Resource Meta-Data Best Practice and Implementation Guide provides general guidance about how an application may use LOM meta-data elements. The IMS Learning Resource XML Binding specification provides a sample XML representation and XML control files (DTD, XSD) to assist developers with their meta-data implementations." Available online from http://www.imsglobal.org/. [Accessed 11 November 2003].

10. Locally, CICERO, the Committee for Institutional Cooperation's Educational Resource Repository project that enables the sharing of digital objects among members is another active digital project gaining momentum. Available online from http://cicero.tlt.psu.edu/. Both CICERO and ANGEL support IMS.

11. View the home page of the IMS project at http://www.imsproject.org/. [Accessed 11 November 2003].

12. VIUS data element definitions are available online at http://www.libraries.psu.edu/vius/ reports/VIUSElementDescription.doc. [Accessed 11 November 2003].

13. The IMS Learning Resource Meta-data Best Practices and Implementation Guide are available online at http://www.imsproject.org/metadata/mdbestv1p1.html. [Accessed 11 November 2003].

14. VRA Core Categories, Version 3.0, a project of the Visual Resources Association Data Standards Committee, is available online from http://www.vraweb.org/vracore3.htm. [Accessed 11 November 2003]. From the VRA Core: STYLE/ PERIOD

Qualifiers: 1

Style/Period.Style 1

Style/Period.Period 1

Style/Period.Group 1

Style/Period.School 1

Style/Period.Dynasty 1

Style/Period.Movement 1 
Description: A defined style, historical period, group, school, dynasty, movement, etc., whose characteristics are represented in the Work or Image.

Dublin Core: COVERAGE, SUBJECT

15. The hierarchy can best be illustrated by an example expressed in SGML-style tags. The subject term "Landscape Architecture" would be expressed in IMS as

$<$ classification>

$<$ purpose $>$ Discipline $<$ /purpose $>$

$<$ taxonPath>

$<$ source $>\mathrm{LCSH}<$ /source $>$

$<$ taxon>

$<$ entry $>$ Landscape Architecture</entry $>$

$</$ taxon $>$

$</$ taxonPath $>$

$<$ classification $>$

This identifies landscape architecture as a discipline (subject) taken from the Library of Congress subject headings (LCSH) list.

16. One could foresee a useful purpose in permitting students in a class section to associate their own descriptive terms with a learning object accessed through a course management system. Such user-supplied metadata could be persistent through a semester and then lapse or remain confined to that section's view of the resources (perhaps even student by student). Such an application of user-supplied metadata approaches the concepts captured in the term "democratic indexing," as described in Brown et al, "The Democratic Indexing of Images," New Review of Hypermedia and Multimedia (2): 107-19.

17. Most research on mining image data deals with pattern recognition and provides nothing in the way of content analysis.

18. See W3C's Semantic Web site at http://www.w3.org/2001/sw/. [Accessed 8 October 2003].

19. IMS Global Learning Consortium's VDEX site is located online at http://www.imsglobal. org/vdex/index.cfm. [Accessed 8 October 2003]. 